\title{
CORE LEVEL EXCITATION OF ATOMIC AND MOLECULAR CLUSTERS
}

\author{
E. Rühl, C. Schmale, H.W. Jochims, E. Biller, R. Locht ${ }^{*}$, A.P. Hitchcock ${ }^{\dagger}$, and H. Baumgärtel \\ Institut für Physikalische und Theoretische Chemie, Freie Universität Berlin, Takustr. 3, D-1000 Berlin 33, F.R. Germany
}

\begin{abstract}
Core level excitation of weakly bonded atomic and molecular species is reported. Excitations into unoccupied orbitals with valence or Rydberg character as well as into the corresponding K- and L-continua are compared with the isolated and condensed species. Core level excitation of clusters is also accompanied with extensive fragmentation of singly and doubly charged clusters. Coincidence techniques such as photoelectronphotoion-coincidences (PEPICO) and photoion-photoion-coincidences (PIPICO) are used to study the photochemical fragmentation pathways of core excited clusters. Kinetic energy releases related to charge separation of cluster dications (Coulomb explosion) are derived from PIPICO spectra. The fragmentation energetics is discussed in relation to cluster dication threshold energies.
\end{abstract}

\section{INTRODUCTION}

The field of core excitation processes of clusters is relatively unexplored. Most studies that have been performed in the energy regime of core level excitation $(\mathrm{E}>150 \mathrm{eV})$ have concentrated on isolated atoms or molecules in the gas phase and condensed species, i.e. solids or adsorbates. ${ }^{1,2}$ Valuable information is obtained from these studies, such as energy positions and relative intensities of resonant processes above or below the corresponding ionization energies. It has been found that the shapes of inner shell absorption spectra of isolated atoms differ from those of the corresponding condensed species. ${ }^{3}$ In contrast to this valence transitions of core excited molecular species $\left(1 \mathrm{~s} \rightarrow \pi^{*}\right)$ show typically the same energy positions in the gas and condensed phase. ${ }^{4}$ However, intensity changes of discrete resonances relative to the corresponding ionization continua are observed. ${ }^{4}$ Recently, we have reported progress in the field of core level excitation of isolated clusters in the gas phase. ${ }^{5}$ It has been shown that in the case of argon clusters the cation yield spectra of mass selected clusters differ considerably from those of the isolated cation in the $\mathrm{L}_{3} / \mathrm{L}_{2}$ excitation regime (240-300 eV). Cluster specific resonances are observed, which are more similar to the absorption spectrum of the solid rather than to the atom. Broad features are observed in the corresponding $\mathrm{L}_{3} / \mathrm{L}_{2}$ continuum, which are assigned as single scattering (EXAFS) processes in clusters. More recent results in this field show that also above the $\mathrm{L}_{1}$ edge similar EXAFS structure is observed (320-500 eV), which gives further confidence in the given assignments. ${ }^{6}$ We have also studied fragmentation pathways of core excited argon cluster dications. ${ }^{7}$ With the photoion-photoion-coincidence (PIPICO) technique it was shown that doubly charged clusters undergo asymmetric fission, leading to series of products, such as monomer cations, dimer cations, and trimer cations in coincidence with cluster cations. The kinetic energy releases arc significantly lower than expected from a simple electrostatic picture» which is in agreement with findings for collision induced fragmentation carbon dioxide cluster dications, where internal exitation has been assumed to be responsible for the low kinetic energy releases. ${ }^{8}$

In this paper we report recent progress in the field of core level excitation in atomic and molecular clusters in the energy regimes of element K- and L-edges: argon 2p (240-280 eV), carbon 1s (270-330 eV), and oxygen 1s (520-570 eV). Besides photoion yield curves of mass selected cluster cations, which provide spectroscopic information, we have also studied fragmentation reactions of core excited cluster monocations and dications. Threshold energies of unstable argon cluster dications are measured, which are used to interpret the energetics of core excited cluster dication decay.

\section{EXPERIMENTAL}

The experiments were performed at the electron storage ring BESSY. The high energy TGM-II beam line allows the dispersion of soft $\mathrm{x}$ rays between $150 \mathrm{eV}$ and $600 \mathrm{eV}$. The energy resolution $(\Delta \mathrm{E})$ is obtained from the full width at half maximum signal of the argon $\left(\mathrm{L}_{3}{ }^{-1}, 4 \mathrm{~s}\right)$ ion signal at $244.390 \mathrm{eV}$. The experiments were performed typically with an energy resolving power $(\mathrm{E} / \Delta \mathrm{E})$ of $300-500$.

\footnotetext{
*permanent address: Départment de Chimie Générale, Université de Liège, Sart-Tilman, B-4000 Liège, Belgium

† permanent address: Department of Chemistry, McMaster University, Hamilton, Ontario, L8S 4M1, Canada
} 
The TGM-7 beam line is used for threshold experiments of direct cluster double ionization. This monochromator disperses synchrotron radiation between $7 \mathrm{eV}$ and $150 \mathrm{eV}$. The experiments are performed with a $950 \mathrm{l} / \mathrm{mm}$ grating with an optimized dispersion range between 20-120 eV. Higher order light and stray light was filtered by an aluminum foil.

The experimental setup shown in Fig. 1 is similar to that reported earlier. ${ }^{5}$ A time of flight mass spectrometer (TOF-MS) is used for cation detection. The design of the mass spectrometer involves conventional Wiley/McLaren space focussing. Under typical experimental conditions a mass resolution $(\mathrm{M} / \Delta \mathrm{M})$ of 125 is obtained for $\operatorname{Ar}^{+}(\mathrm{m} / \mathrm{z}=40)$. The spectrometer is operated with moderate acceleration fields in the ionization region (100 to $200 \mathrm{~V} / \mathrm{cm})$.

Opposite to the TOF-MS a threshold photoelectron spectrometer is mounted. This device can be replaced by a total yield detector.

With this setup we have measured mass spectra at resonant excitation energies, total cation and electron yield curves, photoionization efficiency curves of mass selected cations, threshold photoelectron (TPES) spectra, photoelectron-photoion-coincidence (PEPICO) spectra, and PIPICO spectra. Mass spectra were recorded by the use of pulsed extraction of cations into the mass spectrometer, whereas PEPICO and PIPICO spectra were obtained with static voltages. Further experimental details are given in ref. 7.

Fig. 1. Experimental setup

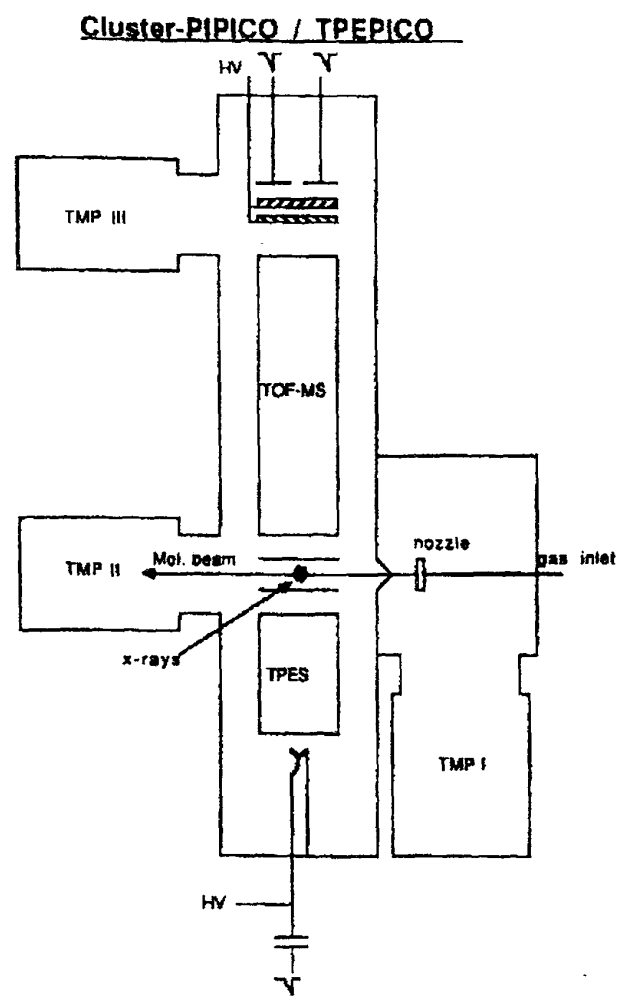

The stagnation conditions for the supersonic expansion are similar to those reported earlier. ${ }^{5,7}$ We have extended the stagnation temperature range by cooling the gas inlet line and the nozzle down to $90 \mathrm{~K}$ with liquid nitrogen. From scaling laws and correlations to average cluster sizes we expect for $\mathrm{T}_{0}=300 \mathrm{~K}$ an average cluster size $(\overline{\mathbf{N}})$ of $2\left(\mathrm{p}_{0}=2\right.$ bar) to $10\left(\mathrm{p}_{0}=5\right.$ bar), depending on the expansion conditions. ${ }^{5}$ Kinetic energy releases (KER) for charge separation in cluster dications are calculated according to the formulas given in ref. 9. The samples were of commercial quality and were used without further purification. 


\section{RESULTS AND DISCUSSION}

\section{ATOMIC CLUSTERS}

Fig. 2 shows the photoion-photoion-coincidence (PIPICO) spectrum of argon clusters recorded at 248 $\mathrm{eV}$. This energy corresponds to $3 \mathrm{~d}(3 / 2)$ exciton state in clusters and the solid. ${ }^{3,6,7}$ Three series of charge separation product channels are identifed: $\mathrm{Ar}^{+} / \mathrm{Ar}_{\mathrm{n}}{ }^{+}, \mathrm{Ar}_{2}{ }^{+} / \mathrm{Ar}_{\mathrm{n}}{ }^{+}$and $\mathrm{Ar}_{3}{ }^{+} / \mathrm{Ar}_{\mathrm{n}}{ }^{+}$. These series of charge separation show that asymmetric fission of argon cluster dications is dominant. The signal at $\Delta \mathrm{t}=0$ is shown to be not due to symmetric charge separation processes. However, forthcoming photoelectron-photoion-photoion-coincidence experiments will unequivocally show the fraction of symmetric versus asymmetric charge separation in core excited argon clusters.

The series of coincidences involving cation pairs of dimers and clusters is stronger than the other ones. This is consistent with the high stability of the argon dimer cation. Its formation is a dominant process in single ionization of argon clusters. Recent experimental results from mass resolved molecular beam scattering show that small neutral clusters form preferentially monomer and dimer cations after electron impact ionization. ${ }^{10 a}$ This is in agreement with photofragmentation studies of mass selected cluster cations, where for species smaller than $\mathrm{Ar}_{15}{ }^{+}$the major fragments $\mathrm{Ar}_{2}^{+}$and $\mathrm{Ar}_{3}{ }^{+}$are detected. ${ }^{10 \mathrm{~b}}$ From photoelectron spectra of argon cluster beams and DIM calculations the chromophors $\mathrm{Ar}_{3}{ }^{+}$(for small clusters) and $\mathrm{Ar}_{13}{ }^{+}$(for large clusters) are proposed. ${ }^{11}$

Asymmetric fission of molecular cluster dications has been studied with electron impact ionization techniques. It is reported that more than $90 \%$ of the mass is retained in the observed fagments, ${ }^{8}$ whereas stable doubly charged argon clusters undergo a loss of one or two single atoms, where no Coulomb explosion is observed. $^{12}$

Fig. 2. (a) Photoion-photoion-coincidence (PIPICO) spectrum of argon clusters at 248 eV excitation energy, (b) shows a detailed view of the $\mathrm{Ar}^{+} / \mathrm{Ar}_{2}{ }^{+}$signal. The energy scale refers to the two body dissociation $\mathrm{Ar}_{3}{ }^{++} \rightarrow \mathrm{Ar}^{+} / \mathrm{Ar}_{2}{ }^{+}$. Experimental conditions: $\mathrm{p}_{0}=4.7$ bar, $\mathrm{T}_{0}=215 \mathrm{~K}$.

(a)

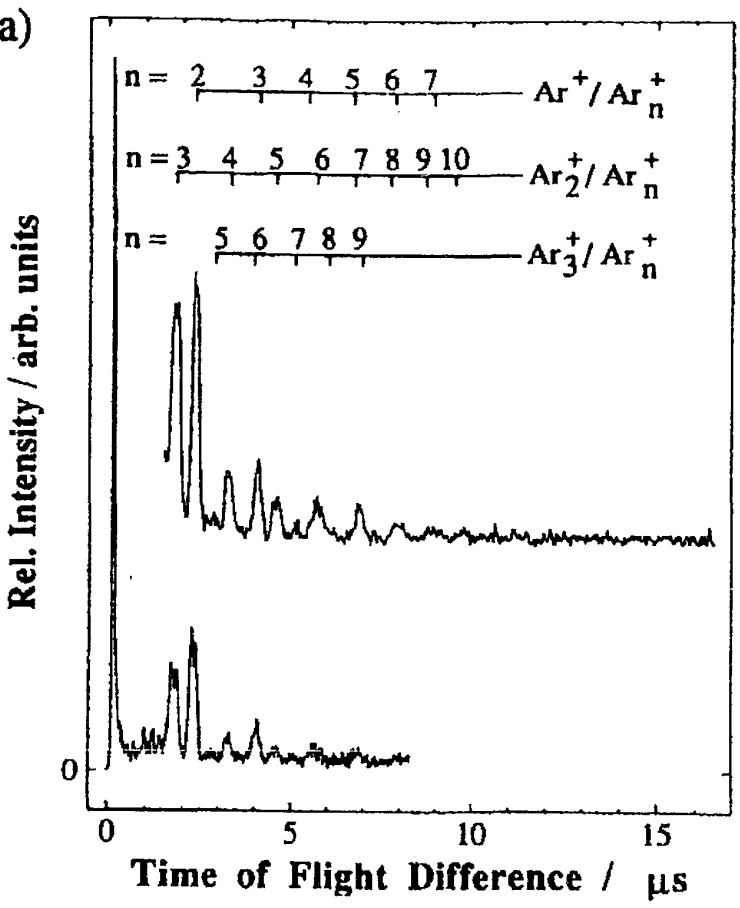

(b)

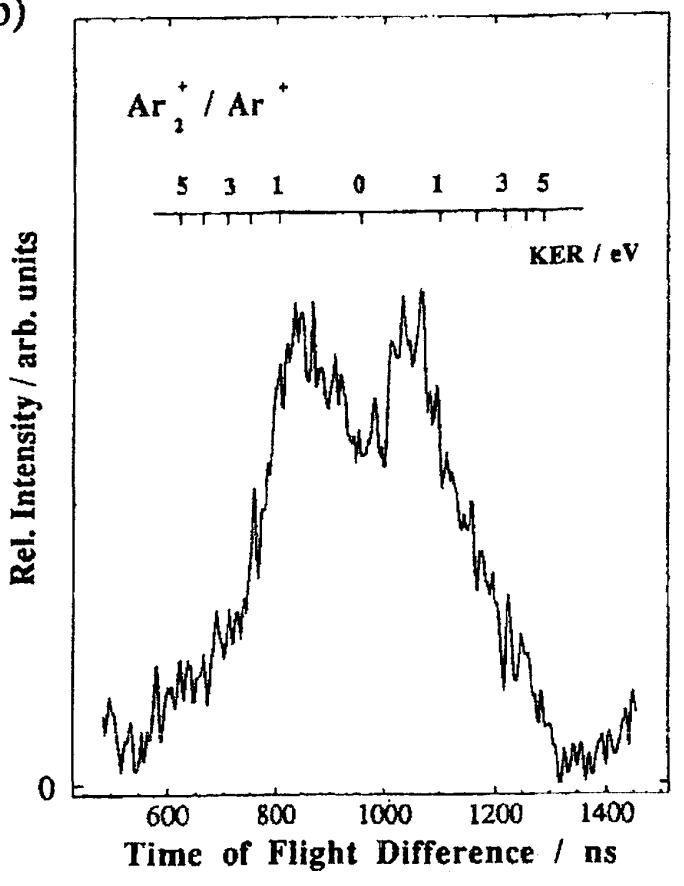

Fig. 2 (b) shows a detailed view of the $\mathrm{Ar}^{+} / \mathrm{A}_{\mathrm{r} 2}{ }^{+}-\mathrm{PIPICO}$ signal. From the width of the coincidence signal one obtains the corresponding kinetic energy release (KER), which is indicated in Fig. $2 b$ for a two body dissociation reaction $\left(\mathrm{Ar}_{3}{ }^{++} \rightarrow \mathrm{Ar}^{+} / \mathrm{Ar}_{2}{ }^{+}\right)$. Various other charge separation reactions involving three body dissociations and evaporation of neutrals are discussed in ref. 7. In a purely electrostatic picture the KER corresponds to a charge separation distance. For the widest portion of the PIPICO signal a charge separation distance of 3.8(3) $\AA$ is calculated. This value is in agreement with the value found from EXAFS in argon clusters 


\section{(3.9(1) $\AA),{ }^{5}$ the solid $(3.75 \AA)$, and matrices $(3.7 \AA) .{ }^{13}$}

The time separation of both maxima of the PIPICO signal corresponds to a KER of $0.7(2) \mathrm{eV}$. It is remarkable that the shape of the PIPICO signal remains nearly unchanged if the average cluster size is decreased.

A likely explanation for the low KER's can be found by considering a complex charge separation mechanism in cluster dications: According to the double ionization model for solids a two step process leads to double ionization, where first step is the photoionization of one atom within the cluster followed by subsequent electron impact ionization of a neighboring atom. Immediate Coulomb exposion will result in a high KER, which accounts for the widest part of the PIPICO signal. As a competing process charge separation within the clusters prior to Coulomb explosion is likely. Some of the total energy in the cluster dication may be consumed by evaporation of neutrals or can be stored as internal energy of the singly charged fragments. Similar results are reported by Gotts and Stace for collision induced fragmentation of carbon dioxide cluster dieations. ${ }^{8}$ The difference between the predicted KER's for Coulomb repulsion and the experimental results are interpreted in terms of simultaneous internal excitation.

In an oversimplified picture of purely electrostatic charge separation the low KER can be understood in terms of medium sized cluster dications, such as $\mathrm{Ar}_{7}{ }^{++}$to $\mathrm{Ar}_{8}{ }^{++}$, where a charge separation distance of $20.5 \AA$ appears to be in a reasonable range, if non-compact cluster structures are assumed to exist prior to charge separation.

Related to the KER's in clusters is the question of energy position of the dissociating dication state. The asymptote of the $\mathrm{Ar}^{+} / \mathrm{Ar}_{2}^{+}$product channel is $30 \mathrm{eV}$ as calculated from thermodynamical reference data. ${ }^{14} \mathrm{With}$ the experimental KER of $3.8 \mathrm{eV}$ one obtains $33.8 \mathrm{eV}$ as a lower limit for the dissociating dication state of $\mathrm{Ar}_{3}{ }^{++}$.

Experimental efforts have been made to measure thresholds of unstable cluster dications in order to correlate the above estimated energy of dissociative dication states with experimental data. Since small cluster dications are unstable we have used the PIPICO-technique to investigate both the thresholds of the $\mathrm{Ar}^{+} / \mathrm{Ar}_{2}^{+}$and $\mathrm{Ar}_{2}{ }^{+} / \mathrm{Ar}_{3}{ }^{+}$PIPICO signals as a function of average cluster size and photon energy. It is observed that the thresholds of both product channels depend on the average cluster size. The results are listed in Tab. I:

Table I: Threshold energies for double ionization in atomic, clustered and solid argon. The average cluster sizes are estimated to the references listed in ref. 5. The error limits for cluster threshold determination are $0.3 \mathrm{eV}$.

\begin{tabular}{cccc}
\hline species & & threshold energy/eV & reference \\
\hline atom & $\overline{\mathbf{N}}=3$ & 43.4 & $15 \mathrm{a}$ \\
cluster & 36.0 & this work \\
& $\overline{\mathbf{N}}=4$ & 35.5 & this work \\
& $\overline{\mathbf{N}}=6$ & 35.0 & this work \\
& $\overline{\mathbf{N}}=10$ & 33.5 & this work \\
& & 32.8 & this work \\
Ar $_{101}$ & & 32 & $15 \mathrm{~b}$ \\
solid & 20 & & $15 \mathrm{c}$ \\
\hline
\end{tabular}

The data in Tab. I show that the double ionization thresholds of the solid and the atom differ by nearly $15 \mathrm{eV}$. The value for solid argon $(28.6 \mathrm{eV})$ is obtained from recent photoelectron-photoelectron-coincidence measurements, which is in good agreement with the double ionization energy of the solid. ${ }^{15 \mathrm{c}}$ From electron impact mass spectrometry a threshold for the stable cluster dication $\mathrm{Ar}_{101}{ }^{++}$is reported. ${ }^{15 \mathrm{~b}}$ The thresholds of cluster dications undergoing Coulomb explosion show values that fall between the stable cluster dication $\left(\mathrm{Ar}_{101}{ }^{++}\right)$and the atomic dication. The estimated lower limit of the $\mathrm{Ar}_{3}{ }^{++}$dissociative state $(33.8 \mathrm{eV})$ agrees well with the appearance energies of small cluster dications $(\overline{\mathbf{N}}<6)$.

\section{MOLECULAR CLUSTERS}

Fig. 3 shows photoionization efficieny curves of $\mathrm{CO}$ and its dimer in the energy regime of the carbon $\mathrm{K}$-edge as well as the $\mathrm{C}^{+}$and dimer spectra at the oxygen K-edge. All spectra are dominated by the $\mathrm{C}(1 \mathrm{~s}) \rightarrow \pi^{*}$ transition $(287.4 \mathrm{eV})$, and $\mathrm{O}(1 \mathrm{~s}) \rightarrow \pi^{*}$ transition $(534.11 \mathrm{eV})$, respectively. 
Fig. 3. Photoionization efficiency curves of (a) $\mathrm{CO}^{+}$and $(\mathrm{CO})_{2}{ }^{+}$in the regime of the carbon $\mathrm{K}$-edge and (b) $\mathrm{C}^{+}$ and $(\mathrm{CO})_{2}{ }^{+}$in the regime of the oxygen $\mathrm{K}$-edge. Experimental conditions: $p_{0}=4.7$ bar, $T_{0}=170 \mathrm{~K}$.
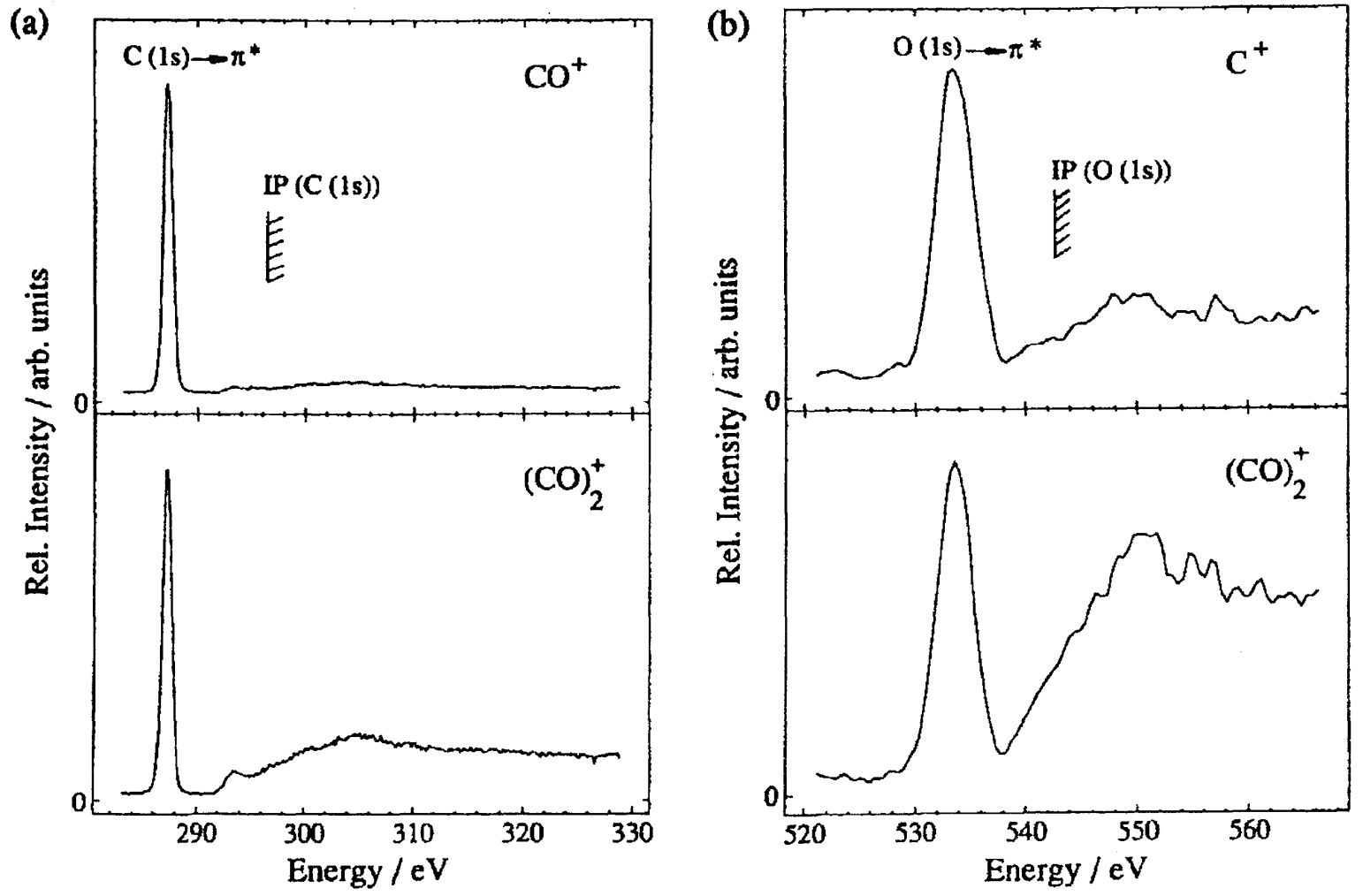

In contrast to the cluster size dependent energy positions of Rydberg states below the argon $2 \mathrm{p}$ edges, we do not observe energy shifts for this valence transition in clusters. This is in agreement with photon stimulated desorption of solid carbon monoxide, where no shift relative to the gas phase spectrum is observed. ${ }^{4}$ The most interesting difference in spectral shape is the decreased intensity of both $1 \mathrm{~s} \rightarrow \pi^{*}$ bands relative to the Kcontinuum intensities and changes in spectral shape in both Rydberg regimes. The $\mathrm{x}$ ray absorption spectrum of $\mathrm{CO}$ shows under high resolution conditions evidence for several Rydberg series below the carbon K-ionization continuum. ${ }^{16}$ In the dimer spectrum we find an onset of intensity at $292 \mathrm{eV}$ without any fine structure, a shoulder at $293 \mathrm{eV}$, and an increase in intensity up to $305 \mathrm{eV}$, which corresponds to the energy of the $\mathrm{C}(1 \mathrm{~s}) \rightarrow \sigma^{*}$ transition. The spectral shape of the dimer cation mimicks that of cations desorbing from the solid (photon stimulated desorption (PSD)). ${ }^{4}$ The ion yield curve of $\mathrm{CO}^{+}$shows also weak contributions of cluster ion fragmentation above $292 \mathrm{eV}$. In gas phase $\mathrm{CO}$ one finds that there is no parent cation intensity above the carbon $\mathrm{K}$-ionization energy, ${ }^{17}$ since double and multiple ionization dominate this energy regime. This shows that cluster fragmentation leads to $\mathrm{CO}^{+}$as a final product of dissociative ionzation processes. This is also consistent with the PEPICO spectrum of $\mathrm{CO}$ clusters recorded at $305 \mathrm{eV}$, where a considerable $\mathrm{CO}^{+}$signal is observed (cf. Fig. 4a). Another interesting difference of the cluster cation yield is a comparison with coordinated $\mathrm{CO}$ in transition metal carbonyls. ${ }^{18}$ It is found that quenching of Rydberg states is evident as observed for van der Waals clusters of CO. Due to metal $3 \mathrm{~d}$ level backbonding the intensity of the $\mathrm{C}(1 \mathrm{~s}) \rightarrow \pi^{*}$ transtion is decreased in organometallics.

Similar differences in spectral shape are observed for oxygen K-electron excitation, where a considerably stronger K-continuum is observed relative to the $\mathrm{O}(1 \mathrm{~s}) \rightarrow \pi^{*}$ resonance. This is in good agreement with PSD of cations from the solid. ${ }^{4}$ Relative changes in intensity of $\pi^{*}$ resonances vs. K-continuum intensities have been rationalized for the solids in terms of a delayed onset behavior. ${ }^{4}$ These phenomena are consistent with reneutralization or screening effects by the solid. It is interesting to note that the spectral shape of the $\mathrm{C}^{+}$ fragment shows a considerably stronger $\mathrm{O}(1 \mathrm{~s}) \rightarrow \pi^{*}$ resonance compared to the dimer cation. This is consistent with the spectra shown in ref. 17, indicating that $\mathrm{C}^{+}$is a product of unclustered $\mathrm{CO}$ fragmentation. 
Fig. 4. (a) Photoelectron-photoion-coincidence (PEPICO) spectrum and (b) photoion-photoion-coincidence (PIPICO) spectrum of carbon monoxide clusters at $305 \mathrm{eV}$ excitation energy. Experimental conditions: $p_{0}=4.7$ bar, $T_{0}=170 \mathrm{~K}$.
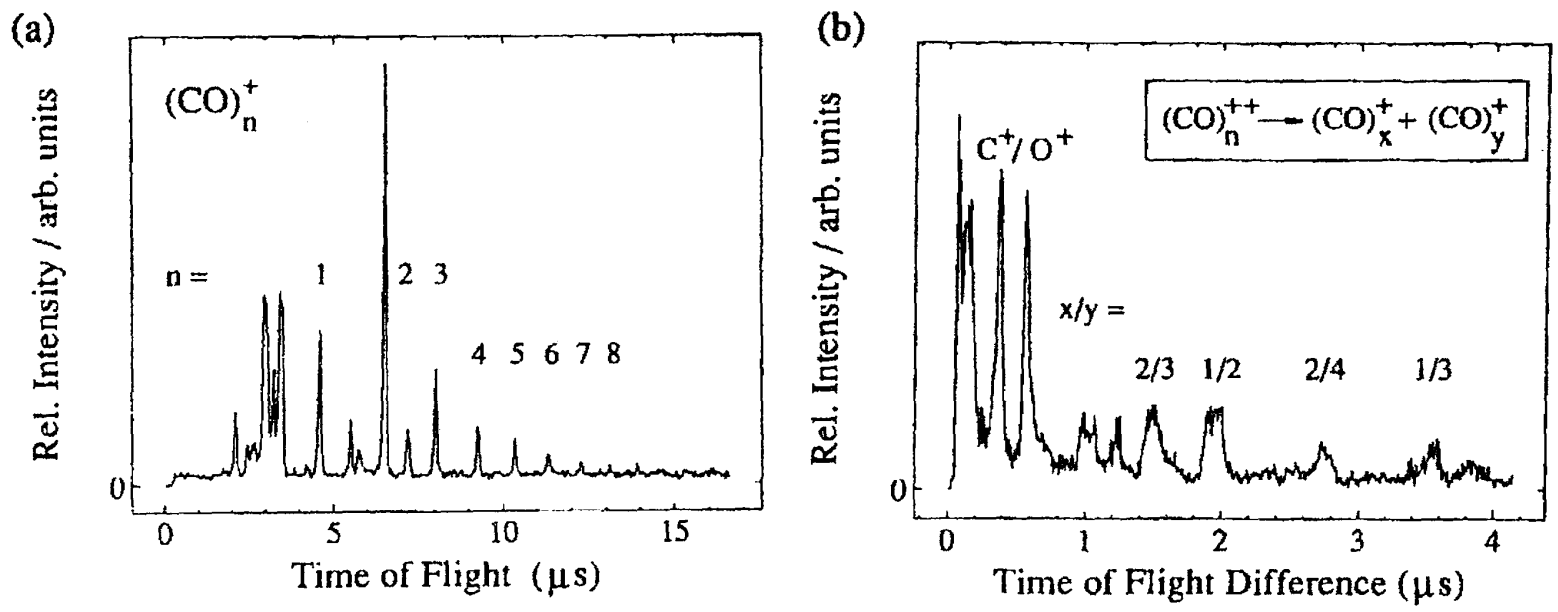

The photochemical behavior of carbon monoxide clusters are studied with the PEPICO and PIPICO techniques. The PEPICO spectrum of CO clusters is shown in Fig. 4a. The dominant peak in the spectrum is the dimer cation, which is one of the preferred fragmentation products of $\mathrm{CO}$ cluster dications, as deduced from the width of the PEPICO signal. Besides the dimer signal we find a relative strong monomer intensity as well as singly or multiply charged atomic fragments. The latter ones show unchanged intensity upon clustering, indicating that they are not involved in cluster dissociation pathways.

The PIPICO spectrum of CO clusters shows besides the well-known molecular charge separation channels $\left(\mathrm{C}^{+}+\mathrm{O}^{+}\right)$several coincidences corresponding to cluster dication decays. Most products involve asymmetric charge separation into entire molecular and cluster units. The widest portion of the $\mathrm{CO}^{+} /(\mathrm{CO})_{2}{ }^{+}$ PIPICO channel corresponds to a KER of $2.6(3) \mathrm{eV}$ for a two body dissociation, whereas the peak maximum gives $0.9(2) \mathrm{eV}$. In terms of charge separation distances in an electrostatic (Coulomb explosion) picture this corresponds to $5.5 \AA$ and $17 \AA$, respectively. The lower value is twice the molecular diameter, ${ }^{19}$ which is maximum charge separation distance in a dimer. The higher value corresponds to a low KER which is probably due to internal excitation of the cluster fragments, as discussed for argon clusters.

\section{ACKNOWLEDGEMENTS}

Financial support by the Bundesminsterium für Forschung und Technologie is gratefully acknowledged. R.L. acknowlegdes financial support by the European Community. A.P.H. acknowledges NATO for a travel grant. We thank H. Schmelz for technical assistance.

\section{REFERENCES}

1 A.P. Hitchcock, J. El. Spectrosc. Relat. Phenom. 25, 245 (1982), and update 1990 (A.P. Hitchcock, private communication); A.P. Hitchcock Physica Scripta T31, 159 (1990)

2 J. Stöhr and D. A. Outka, J. Vac. Sci. Tech. A 5, 919 (1987); D. Outka and J. Stöhr, J. Chem. Phys. 88, 3539 (1989); J. Stöhr, NEXAFS Spectroscopy (Springer Series in Surface Science, Springer, Berlin, 1991)

3 R. Haensel, G. Keitel, N. Kosuch, U. Nielsen, and P. Schreiber, J. de Physique C 4, 236 (1971)

4 R.A. Rosenberg, P.J. Love, P.R. LaRoe, V. Rehn, and C.C. Parks, Phys. Rev. B 31. 2534 (1985)

5 E. Rühl, H.W. Jochims, C. Schmale, E. Biller, A.P. Hitchcock and H. Baumgärtel, Chem. Phys. Lett. 178, 558 (1991), and references therein

6 E. Rühl, C. Schmale, H.W. Jochims, E. Biller A.P. Hitchcock, and H. Baumgärtel, to be published

7 E. Rühl, C. Schmale, H.W. Jochims, E. Biller, M. Simon, and H. Baumgärtel, J. Chem. Phys., in press (1991)

8 N.G. Gotts and A.J. Stace, Phys. Rev. Lett. 66. 21 (1991) 
Published in: Synchrotron Radiation and Dynamic Phenomena (1992), pp. 230-240.

Status: Postprint (Author's version)

9 D.M. Curtis and J.H.D. Eland, Int. J. Mass Spectrom. Ion Proc. 63, 241 (1985)

10 (a) U. Buck and H. Meyer, J. Chem. Phys. 84, 4854 (1986); (b) N.E. Levinger, D. Ray, M.L. Alexander, and W.C. Lineberger, J. Chem. Phys. 89, 5654 (1988) 11 F. Carnovale, J.B. Peel, R.G. Rothwell, J. Valldorf, and P.J. Kuntz, J. Chem. Phys. 90, 1452 (1989)

12 A.J. Stace, P.J. Lethbridge, and J.E. Upham, J. Chem. Phys. 93.333 (1989)

13 W. Niemann, W. Malzfeld, P. Rabe, R. Haensel, and M. Lübcke, Phys. Rev. B 35, 1099 (1987)

14 H.M. Rosenstock, K. Draxl, B.W. Steiner, and J.T. Herron, J. Phys. Chem. Ref. Data 6 (Suppl. 1) (1977); J. Jortner, Ber. Bunsenges. Phys. Chem. 88, 188 (1984)

15 (a) D.M.P. Holland, K. Codling, J.B. West, and G.V. Marr, J. Phys. B, At. Mol. Phys. 12, 2465 (1979); (b) P. Scheier and T.D. Märk, Chem. Phys. Lett. 136, 423 (1987); (c) H.W. Biester, M.J. Besnard, G. Dujardin, L. Hellner, and E.E. Koch, Phys. Rev. Lett. 59, 1277 (1987)

16 M. Domke, C. Xue, A. Puschmann, T. Mandel, E. Hudson, D.A. Shirley, and G. Kaindi, Chem. Phys. Lett. 173, 122 (1990), Chem. Phys. Lett. 174, $668(1990)$

17 A.P. Hitchcock, P. Lablanquie, P. Morin, E. Lizon, E. Lugrin, M. Simon, P. Thiry, and I. Nenner, Phys. Rev. A 37,2448 (1988)

18 E. Rühl and A.P. Hitchcock, J. Am. Chem. Soc. 111, 2614 (1989)

19 Gmelins Handbuch der Anorganischen Chemie, "Kohlenstoff Teil C -Licferung 1", (Verlag Chemie, Weinheim, 1970), p. 123

\section{QUESTION}

$\underline{B A E R}$ - I am surprised that you obtained similar bond distances for the $\operatorname{Ar}_{2}$ clusters as for the $\operatorname{Ar}_{\mathrm{n}}$ clusters from your NEXAFS data. What about multiple scattering?

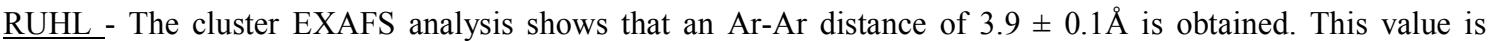

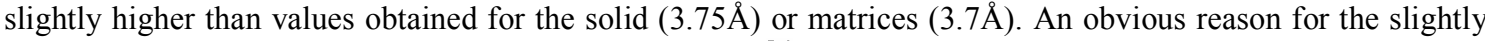
increased Ar-Ar distance in small argon clusters (with $\overline{\mathbf{N}}=10$ ) can be found in the spherical (icosahedral) structure of argon clusters. In contrast to this, the solid is known to exist in a f.c.c. lattice. Further EXAFS experiments in the energy regime of $\operatorname{Ar}(1 \mathrm{~s})$ excitation are needed to confirm the results obtained at the $\operatorname{Ar}(2 p)$ edge $(250 \mathrm{eV})$. The advantage of $\operatorname{Ar}(1 \mathrm{~s})$ - EXAFS will be, that the energy space is not limited as in the case of $\operatorname{Ar}(2 p)$ - EXAFS, where the $\operatorname{Ar}(2 s)$ - edge disturbs the $2 p$ EXAFS above $330 \mathrm{eV}$. Another advantage of $\operatorname{Ar}(1 \mathrm{~s})-$ EXAFS will be that only one threshold energy has to be considered in the EXAFS analysis. Our EXAFS analysis has shown, that the oscillations that are observed in photoion yield curves of argon clusters above the $2 \rho$-and $2 \mathrm{~s}$ edges can be described with a single scattering mode, whereas the resonance centered at approximately $256 \mathrm{eV}$ is probably due to multiple scattering.

DUJARDIN - Experimental question about the PIPICO curves you showed. They have a non zero background. So it seems that the false coincidences have not been substituted. Could this change some of the results ?

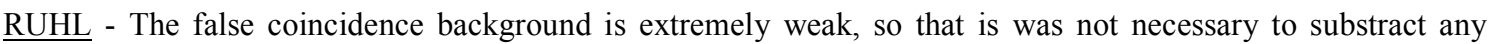
background. Therefore, we do not think the results are affected by false (or random) coincidences.

MENZEL - Two questions related to the comparison of clusters and solids :

1. In solid Argon, surface and bulk $2 p$ resonances can be distinguished by using the detection of different ion signals such as Ar+ and $\mathrm{Ar}_{2}{ }^{+}$(see e.g. D. Menzel, Appl. Phys., 1990 and work cited there) or by detecting decay electrons at different exit angles (G. Rocker et al., to be published in Phys. Rev. B). Can a similar distinction be made in the case of clusters?

2. Again in solid Argon, evidence for a bi-exciton excitable by electrons (P. Feulner et al., PRL June 1991) or even by simple photons (P. Feulner et al., to be published) has been found. Is there any evidence that such an excitation also exists in Ar clusters?

\section{RUHL -}

1. Different cation channels will not give a distinction between different processes. However if the beam expansion conditions are changed, the NEXAFS structure at the $\operatorname{Ar}(2 \mathrm{p})$-edges changes its shape independent on the cation channel. We have analyzed the different components of spectral features in the energy regime of the 4s Rydberg/exciton state (244-246eV) using a total electron detection method (E. Ruhl et al., to be published). When the average cluster size is changed we observe how the individual contributions of small clusters (surface excitons), medium sized clusters, and large clusters (bulk excitons) change their relative intensity.

2. We have found no evidence of these processes in clusters so far.

WONG - Was the Fourier transform of the Ar EXAFS phase corrected to give the Ar-Ar distance you quoted ? If so, which (whose) phase shift value did you use?

RUHL - The EXAFS analysis was done by the use of Mc Kale parameters. Further details can be found in: E. Ruhl et al., Chem. phys. lett. 178, 558 (1991). 